$\begin{array}{cl}\text { Türkiye Tarımsal Araştırmalar Dergisi } & \text { Turk J Agric Res } \\ \text { dergipark.gov.tr/tutad } & \text { 2017, 4(1): 59-64 } \\ \text { O TÜTAD } & \text { ISSN: 2148-2306 } \\ \text { e-ISSN: 2528-858X } & \text { doi: 10.19159/tutad.300702 }\end{array}$

\title{
Screening of Oryza sativa L. for Hpt Gene and Evaluation of Hpt Positive Samples Using Houba Retransposon-Based IRAP Markers
}

\author{
Gözde YÜZBAŞIOĞLU, Sevgi MARAKLI*, Nermin GÖZÜKIRMIZI \\ İstanbul University, Faculty of Science, Department of Molecular Biology and Genetics, İstanbul, TURKEY
}

\begin{tabular}{ll}
\hline Received: 20.09.2016 & Accepted: 08.03.2017 \\
\hline${ }^{*}$ Corresponding author: sevgi.marakli@istanbul.edu.tr &
\end{tabular}

\begin{abstract}
Increasing world population needs to enhance agricultural production because of food starvation. Genetically modified organism (GMO) is a way to solve this problem. During gene transfers, DNA is inserted into a plant's genome in a random way. This produces spontaneous genetic changes with movement of transposable elements, and even increases variations. Houba was described as one of the active retrotransposons in rice. The aim of this study was to screen rice samples collected from Turkey, and analyse Houba retrotransposon movements with IRAP technique in transgenic ones and their controls. For this purpose, 71 different rice seeds obtained from different regions of Turkey were used for GMO analysis. All samples were screened by real time PCR to test cauliflower mosaic virus (CaMV) 35S promoter (P-35S) regions, T-NOS (nopaline synthase terminator) regions, figwort mosaic virus (FMV) regions, bar, pat and Crylab/ac, and hpt (hygromycin resistance) genes. Hpt gene was identified in 6 samples as a result of real time PCR analysis. These 6 transgenic samples with their controls were used for IRAP-PCR analysis and 0-56\% polymorphism ratios were observed in analysed samples. This study is one of the first detailed experimental data of transgenic Oryza sativa L. samples in terms of retrotransposon-based variation.
\end{abstract}

Keywords: Genetically modified organism, Houba, IRAP, Real-Time PCR, rice

\section{Introduction}

Plant transformation technology has become an important tool for improving plants especially crop species with desirable traits (Barampuram and Zhang, 2011). Genetically modified organism (GMO) is described as an organism produced from applications of gene transfer methods. The first commercial GMO tomatoes were obtained in 1996 (The Flavor Savor TM). In 2015, agricultural biotech products were grown in 179.7 million hectares of land in the world (James, 2015). These developments have been brought the concerns of adverse effects of GMOs on human, animal health and environment. These concerns have highlighted the concept of biosecurity. For this reason, many countries set official regulations to the label of GMOs and GMO-derivative foods (Matsuoka et al., 2000; Anonymous, 2003; Miraglia et al., 2004; Vijayakumar et al., 2009). Turkey is one of these countries that published a biosafety law in 2010 (Anonymous, 2010a). According to this law, essentially modified crops and GMOs on the market are forbidden for use in infants- and young children-supplementary foods. For this reason, it is obligated to determine GM and GM derivative materials in food and feed products (Anonymous, 2010b). On the other hand, GM plants including Bt11, GA21, NK603, DAS1507, DAS59122, MON89034, MON40-3-2 (GTS-40-3-2), MON89788, A2704-12 have been accepted for using as feed (Anonymous, 2011a, 2011b).

Different methods are used to identify the GMOs. DNA-based methods are listed as GM screening; PCR, nested PCR, microarray and protein-based methods biosensor via ELISA (Gryson, 2010; Cheema et al., 2016; Turkec et al., 2016). Moreover, one of the most useful methods for GM screening is real-time PCR (Gryson, 2010). PCR-based detection can be used in different categories (Holst-Jensen et al., 2003). In the first category, screened regions are specific elements like promoter, terminator sequences as 
CaMV, nopaline synthase terminator or genes encoding the resistance to antibiotics used as markers in selections. In the second category, detection is carried out by identification of specific-genes such as bar, pat and CryIA(b). In category 3 , junctions between promoter and genes, and in category 4 , junction between gene and its integration locus are targeted for PCR amplification. The last one is also called eventspecific which has been demonstrated in plants such as rice, wheat, and maize (Barroso et al., 2015; Zhang et al., 2015; Rao et al., 2016).

There are different DNA-based molecular markers to analyse genome dynamics, polymorphism, and even evolution in plants (Kaya et al., 2013; Poczai et al., 2013; Cakmak et al., 2015). The ubiquity, abundance, dispersion, and dynamism are LTR retrotransposons' characteristics in plant genomes. Therefore, these mobile elements are commonly studied as a molecular marker (Kalendar and Schulman, 2006; Poczai et al., 2013). IRAP (Inter-Retrotransposon Amplified Polymorphism) is one of them. In this method, PCR primers are designed as an outward direction from the conserved sequences of LTR. Therefore, internal regions between two LTRs or solo LTRs (without retrotransposon) are amplified (Kalendar et al., 1999). Transposons also cause different phenotypes; for example, in 4,000 transposon-insertion lines detected, about 140 lines showed a visible mutant phenotype (Kuromori et al., 2006). The first step for successful variation analysis with retrotransposons is to determine plant-specific retrotransposons. Our group recently described Houba retrotransposon as a good variation analyser in rice (Yuzbasioglu et al., 2016).

In this presented work, 71 rice samples were collected from several markets and Trakya Agricultural Research Institute in Turkey. CaMV $35 \mathrm{~S}$ promoter regions, T-NOS regions, FMV regions, bar, pat and Crylab/ac and hpt genes were analysed to identify transgenics in all these samples by real-time PCR. In addition, genetically modified seeds with their non-transgenic controls were compared with IRAP-PCR by using Houba retrotransposon to determine variation.

\section{Materials and Methods}

\subsection{Samples}

71 rice samples were collected from different regions in Turkey. GM rice samples used for positive control were supplied from Assist. Prof. Dr. A. Akbudak from Akdeniz University.

\subsection{DNA extraction}

Genomic DNAs were isolated from rice samples according to Pervaiz et al. (2011) protocol. The quantities of DNAs were measured by Nanodrop (Thermo Scientific, 2000c) and qualities were controlled in $1 \%$ agarose gel electrophoresis.

\subsection{GM detection}

Primers and probes of $h p t$ gene were designed using GenBank (accession number K01193.1) and GenScript Real-time PCR (TaqMan) primer design programme (https:/www.genscript.com/ssl-bin/ app/primer). The nucleotide sequences of primers and probes were shown in Table 1.

\subsection{PCR conditions for GM analysis}

PCR was carried out in a thermal cycler (Agilent Mx300P). PCR assays were performed in a final volume of $25 \mu \mathrm{l}$ with $12.5 \mu \mathrm{l}$ of PCR mix and $7.5 \mu \mathrm{l}$ Oligo mix and $100 \mathrm{ng}$ of genomic DNA for 35S, NOS, FMV, bar, pat and Crylab/ac screening (Eurofins). In hpt PCR assays for monitoring, the reaction mixture $(25 \mu \mathrm{l})$ contained 12.5 $\mu \mathrm{l}$ of PCR master mix (Agilent Technologies), $200 \mathrm{mM}$ of each primer and probes and $100 \mathrm{ng}$ of genomic DNA. PCR conditions were as follows: initial denaturation at $94^{\circ} \mathrm{C}$ for 10 minutes, 45 cycles of denaturation at $95{ }^{\circ} \mathrm{C}$ for 15 seconds, annealing at $60^{\circ} \mathrm{C}$ for 1 minutes, for $h p t$ gene screening and $94^{\circ} \mathrm{C}$ for 10 minutes, 45 cycles of denaturation at $95^{\circ} \mathrm{C}$ for 15 seconds, annealing at $60^{\circ} \mathrm{C}$ for 1.5 minutes for $35 \mathrm{~S}$, NOS, FMV, crylab/ac, bar and pat screening.

\subsection{IRAP analysis}

After GM detection, transgenic rice samples' gDNAs were used for IRAP-PCR analysis to investigate Houba retrotransposon movements.

Table 1. The Hpt gene primers' and probe's nucleotide sequences

\begin{tabular}{ll}
\hline Hpt $R$ & 5'-ATGCAAAGTGCCGATAAACA-3' \\
Hpt $L$ & 5-'ATGTCCTGCGGGTAAATAGC-3' \\
Hpt $P$ & 5'-FAM- TGCGCCGATGGTTTCTACAAAGATC-TAMRA-3' \\
\hline
\end{tabular}


Primer sequences designed by using IDT (Integrated DNA Technologies) were shown in Table 2. IRAP-PCR was performed in a total volume of $20 \mu \mathrm{L}$, containing $20 \mathrm{ng}$ template DNA, 10 nmol forward and reverse primers and SapphireAmp Fast PCR Master Mix (Takara, RR350A). Primer dimer or other contaminations were checked by using no template control (negative control). In this control, the PCR contents were the same as in IRAP-PCR, but without template (water was used instead of template). The PCR conditions were as follows: initial denaturation at $95{ }^{\circ} \mathrm{C}$ for 2.5 minutes, followed by 30 cycles at $94{ }^{\circ} \mathrm{C}$ for 30 seconds, 51 ${ }^{\circ} \mathrm{C}$ for 30 seconds, $72{ }^{\circ} \mathrm{C}$ for 2.5 minutes and the reaction was completed with a cycle of final extension at $72{ }^{\circ} \mathrm{C}$ for 7 minutes. The PCR products were resolved in a $8 \%$ polyacrylamide (29:1 Acrylamide:Bis) gel electrophoresis (BioRad, Protean II xi Cell) at $150 \mathrm{~V}$ for $8 \mathrm{~h}$ in $1 \mathrm{X}$ TBE buffer $(\mathrm{pH}=8.0)$. A molecular weight marker (Thermo Scientific, SM0331) was also loaded to determine the sizes of the PCR fragments. After, the gel was visualised on UV transilluminator, photographed and used for data analysis.

\subsection{Data analysis}

The well-resolved bands were scored as a binary value, ' 1 ' for presence and ' 0 ' for absence of bands. The binary matrix $(1 / 0)$ was used to calculate the similarity using Jaccard's coefficient (Jaccard, 1908).

Table 2. The Houba retrotransposon primer sequences used for IRAP analysis

\begin{tabular}{ll}
\hline Houba-F & 5'-CTTCGAGTGGGCTAAGGCCC-3' \\
Houba-R & 5'-GTTTCGACCAAGCAGCCGGTC-3' \\
\hline
\end{tabular}

\section{Results}

\subsection{GM analysis}

In this study, rice samples were investigated for GM elements of 35S, NOS, FMV elements and also bar, pat, crylab/ac and hpt. As a result of analysis, no PCR amplification observed in $35 \mathrm{~S}$, NOS, FMV and crylab/ac, bar and pat experiments. On the other hand, we found that 6 samples of 71 rice seeds were transgenic because of hpt gene amplification (Figure 1).

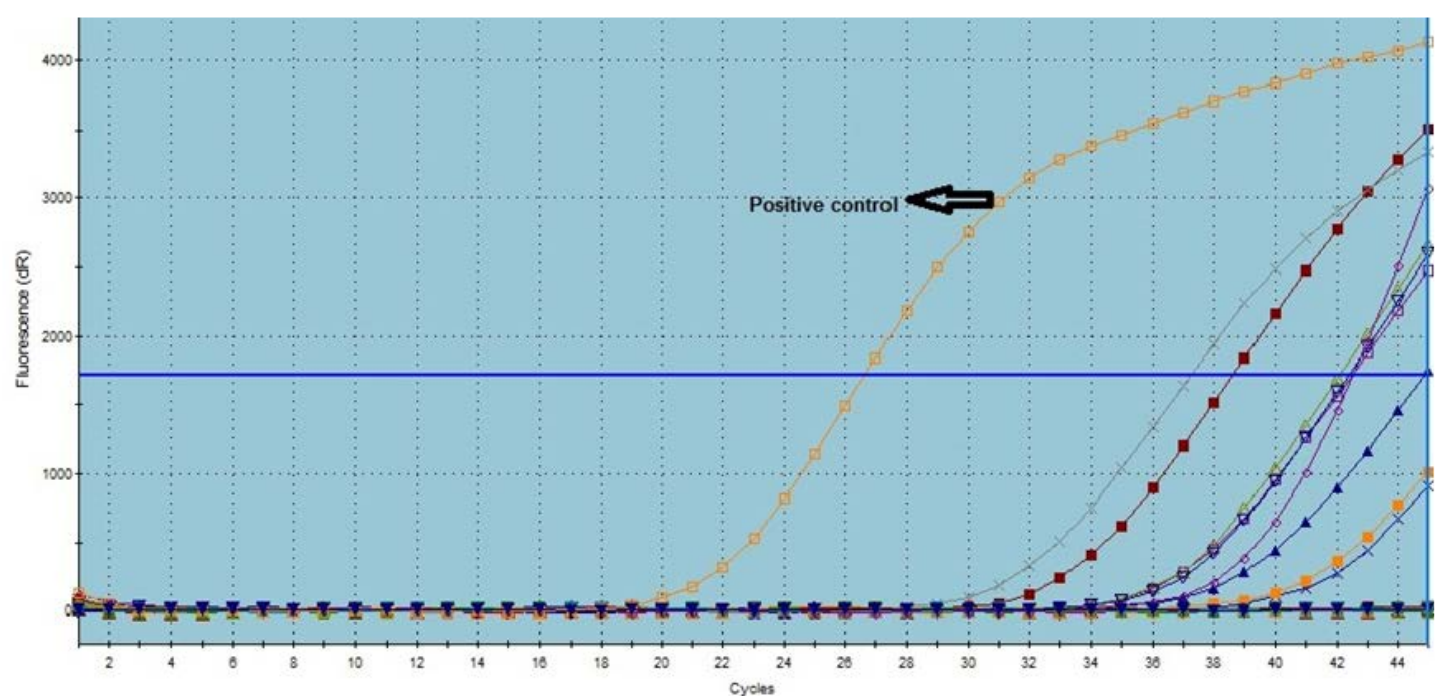

Figure 1. The Hpt gene real time amplification results. Orange line is positive control, other lines are samples

\subsection{IRAP analysis}

After determination of transgenic samples, these seeds with non-transgenic controls were analysed with IRAP marker to determine Houba retrotransposition. Six samples (hygromycin resistant), two control transgenic rice and two nontransgenic rice seeds were investigated using Houba retrotransposon by IRAP-PCR (Figure 2).

Retrotransposon bands showed different profiles among samples with the length between 
250 and $1500 \mathrm{bp}$. IRAP-PCR analysis showed that there were polymorphic bands among samples. Polymorphism rates were 0-56\% among samples. Moreover, polymorphism ratios were $0-25 \%$ for between transgenic samples, $19 \%$ for control transgenic rice and $19 \%$ for non-control transgenic rice (Table 3).

\section{Discussion}

The impact of the insertion of DNA through genetic engineering is probably paralleled by the movement of transposable elements. Transposable elements can be found in the genomes of all plants. Rice genome project results showed that $35 \%$ of rice genome is consist of transposons (TEs). IRAP marker system can be a useful tool for investigating rice breeding (Kalendar et al., 1999). However, a retrotransposon based marker, like IRAP, has not been used for the identification of variation in transgenic rice until now.

Real time PCR is widely used for GMO analysis to identify the presence of most commonly integrated DNA elements in GMOs, and also used event-specific methods provided by the GMO developers (Broeders et al., 2012). In Turkey, the screening process is carried out by Real-Time PCR for only 35S, NOS, and FMV genetic elements. However, there are so many GM events that have different genetic elements rather than 35S, NOS, and FMV. Moreover, hpt gene provides resistance to aminoglycoside antibiotics such as hygromycin B (Van den Elzen et al., 1985). This antibiotic is much more effective than kanamycin for the selection of transformed rice tissues (Christou and Ford, 1995). Zuraida et al. (2013) studied with hpt gene in Agrobacteriummediated genetic transformation of rice and concluded that hygromycin $\mathrm{B}$ is a suitable selection agent and selective marker for genetic transformation. In addition to Agrobacteriummediated transformation, zinc finger nucleases (ZFNs), transcription activator-like effector nucleases (TALENs) and clustered regularly interspaced short palindromic repeats (CRISPR)/CRISPR-associated (Cas) 9 (CRISPR/Cas9) have been commonly used for plant genome editing (Baltes and Voytas, 2015;



Figure 2. Houba retrotransposon variation analysis with IRAP-PCR Transgenic rice: 1, 2, 3, 4, 5, 6. Control transgenic rice: 7, 8. Non-transgenic rice: 9, 10

Table 3. Polymorphism rates of Houba retrotransposon

\begin{tabular}{|c|c|c|c|c|c|c|c|c|c|c|}
\hline & 1 & 2 & 3 & 4 & 5 & 6 & 7 & 8 & 9 & 10 \\
\hline 1 & - & 19 & 19 & 20 & 20 & 13 & 44 & 50 & 7 & 13 \\
\hline 2 & & - & 24 & 25 & 25 & 25 & 40 & 52 & 24 & 29 \\
\hline 3 & & & - & 13 & 13 & 19 & 46 & 56 & 24 & 53 \\
\hline 4 & & & & - & 0 & 7 & 54 & 54 & 29 & 35 \\
\hline 5 & & & & & - & 7 & 50 & 54 & 25 & 31 \\
\hline 6 & & & & & & - & 50 & 50 & 19 & 29 \\
\hline 7 & & & & & & & - & 19 & 40 & 40 \\
\hline 8 & & & & & & & & - & 56 & 50 \\
\hline 9 & & & & & & & & & - & 19 \\
\hline 10 & & & & & & & & & & - \\
\hline
\end{tabular}

Transgenic rice: 1, 2, 3, 4, 5, 6. Control transgenic rice: 7, 8. Non-transgenic rice: 9, 10 
Weeks et al., 2016). Especially CRISPR/Cas9 system has been used for genome editing in major crops such as rice (Zhang et al., 2014; Xu et al., 2015) maize (Feng et al., 2016) and wheat (Shan et al., 2014).

In this study, 35S, NOS, FMV, elements and bar, pat, crylab/ac genes were not detected but $h p t$ gene was found in 6 samples by using Real-Time PCR analysis in total 71 rice seeds collected from different sources. Results showed that the GMO screening process could be modified to increase screening range of genetic elements or genes in Turkey. Houba retrotransposon movements in transgenic rice with their controls were investigated and $0-56 \%$ polymorphism ratios were observed among all samples. In addition, we also found that there were $0-25 \%$ for between transgenic samples, $19 \%$ for control transgenic rice and $19 \%$ for non-control transgenic rice.

In addition to important role of transposon in genome structure and gene functions, information about this issue is still at beginning level (Schnell et al., 2015). In this study, effects of transformation in GM rice on transposon movements were investigated. For this purpose, retrotransposon movements were compared in GM rice and non-GM rice seeds by using Houba retrotransposon specific IRAP-PCR. Results are expected to contribute GMO screening by using hpt gene and determination of transformation results after gene transfers at molecular level.

\section{Acknowledgements}

This work was supported by Scientific Research Projects Coordination Unit of Istanbul University. Project numbers: 40028 and 20366.

\section{References}

Anonymous, 2003. Regulation (EC) No 1830/2003 of the European Parliament and of the Council of 22 September 2003 concerning the traceability and labeling of genetically modified organisms and the traceability of food and feed products produced from genetically modified organisms and amending Directive2001/18/EC, Official Journal L, 268: 00240028 .

Anonymous, 2010a. Turkish Republic Biosafety Law: Law number 5977. Official Journal, 27533/Issue date: 26.03 .2010 .

Anonymous, 2010b. Official Gazette of the Republic of Turkey. Number: 27533. Biosafety Law.

Anonymous, 2011a. Official Gazette of the Republic of Turkey. Number: 27827, Biosecurity Committee Decisions, decision no.1, 2 and 3.
Anonymous, 2011b. Official Gazette of the Republic of Turkey. Number: 28152, Biosecurity Committee Decisions, Decision No. 4-16.

Baltes, N.J., Voytas, D.F., 2015. Enabling plant synthetic biology through genome engineering. Trends in Biotechnology, 33(2): 120-131.

Barampuram, S., Zhang, Z.J., 2011. Recent advances in plant transformation. Plant Chromosome Engineering, pp. 1-35.

Barroso, M.F., Freitas, M., Oliveira, M.B.P.P., De-LosSantos-Alvarez, N., Lobo-Castanon, M.J., DeleureMatos, C., 2015. 3D-nanostructured $\mathrm{Au}$ electrodes for the event-specific detection of MON810 transgenic maize. Talanta, 134: 158-164.

Broeders, S.R.M., De Keersmaecker, S.C.J., Roosens, N.H., 2012. How to deal with the upcoming challenges in GMO detection in food and feed. Journal of Biomedicine and Biotechnology, 2012: 111.

Cakmak, B., Marakli, S., Gozukirmizi, N., 2015. SIRE1 retrotransposons in barley (Hordeum vulgare L.). Russian Journal of Genetics, 51(7): 661-672.

Cheema, H.M.N., Khan, A.A., Khan, M.I., Aslam, U., Rana, I.A., Khan, I.A., 2016. Assessment of Bt cotton genotypes for the CrylAc transgene and its expression. The Journal of Agricultural Science, 154(1): 109-117.

Christou, P., Ford, T.L., 1995. The impact of selection parameters on the phenotype and genotype of transgenic rice callus and plants. Transgenic Research, 4(1): 44-51.

Feng, C., Yuan, J., Wang, R., Liu, Y., Birchler, J.A., Han, F., 2016. Efficient targeted genome modification in maize using CRISPR/Cas9 system. Journal of Genetics and Genomics, 43(1): 37-43.

Gryson, N., 2010. Effect of food processing on plant DNA degradation and PCR-based GMO analysis: a review. Analytical and Bioanalytical Chemistry, 396(6): 2003-2022.

Holst-Jensen, A., Ronning, S.B., Lovseth, A., Berdal, K.G., 2003. PCR technology forscreening and quantification of genetically modified organisms (GMOs). Analytical and Bioanalytical Chemistry, 375(8): 985-993.

Jaccard, P., 1908. Nouvelles recherches sur la distribution florale. Bulletin de la Societe Vaudoise des Sciences Naturelles, 44(163): 223-270.

James, C., 2015. 20th Anniversary (1996 to 2015) of the Global Commercialization of Biotech Crops and Biotech Crop Highlights in 2015. ISAAA Brief No. 51, ISAAA: Ithaca, NY.

Kalendar, R., Grob, T., Regina, M., Suoniemi, A., Schulman, A., 1999. IRAP and REMAP: two new retrotransposon-based DNA fingerprinting techniques. Theoretical and Applied Genetics, 98(5): 704-711.

Kalendar, R., Schulman, A.H., 2006. IRAP and REMAP for retrotransposon-based genotyping and fingerprinting. Nature Protocols, 1(5): 2478-2484. 
Kaya, Y., Yilmaz, S., Gozukirmizi, N., Huyop, F., 2013. Evaluation of transgenic Nicotiana tabacum with deh $\mathrm{E}$ gene using transposon based IRAP markers. American Journal of Plant Sciences, 4(8A): 41-44.

Kuromori, T., Wada, T., Kamiya, A., Yuguchi, M., Yokouchi, T., Imura, Y., Takabe, H., Sakurai, T., Akiyama, K., Hirayama, T., Okada, K., Shinozaki, K., 2006. A trial of phenome analysis using 4000 $D s$-insertional mutants in gene-coding regions of Arabidopsis. The Plant Journal, 47(4): 640-651.

Matsuoka, T., Kawashima, Y., Akiyama, H., Miura, H., Goda, Y., Kusakabe, Y., 2000. A method of detecting recombinant DNAs from four lines of genetically modified maize. Journal of the Hygienic Society of Japan, 41(2): 137-143.

Miraglia, M., Berdal, K.G., Brera, C., Corbisier, P., Holst-Jensen, A., Kok, E.J., Marvin, H.J.P., Schimmel, H., Rentsch, J., Van Rie, J.P.P.F., Zagon, J., 2004. Detection and traceability of genetically modified organisms in the food production chain. Food and Chemical Toxicology, 42(7): 1157-1180.

Pervaiz, Z.H., Turi, N.A., Khaliq, I., Rabbani, M.A., Malik, S.A., 2011. A modified method for highquality DNA extraction for molecular analysis in cereal plants. Genetics and Molecular Research, 10(3): 1669-1673.

Poczai, P., Varga, I., Laos, M., Cseh, A., Bell, N., Valkonen, J.P.T., Hyvönen, J., 2013. Advances in plant gene targeted and functional markers: a review. Plant Methods, 9(6): 1-31.

Rao, J., Yang, L., Guo, J., Quan, S., Chen, G., Zhao, X., Zhang, D., Shi, J., 2016. Development of eventspecific qualitative and quantitative PCR detection methods for the transgenic maize BVLA430101. European Food Research and Technology, 242(8): 1277-1284.

Schnell, J., Steele, M., Bean, J., Neuspiel, M., Girard, C., Dormann, N., Pearson, C., Savoie, A., Bourbanniere, L., Macdonald, P., 2015. A comparative analysis of insertional effects in genetically engineered plants: considerations for premarket assessments. Transgenic Research, 24(1): 117.

Shan, Q., Wang, Y., Li, J., Gao, C., 2014. Genome editing in rice and wheat using the CRISPR/Cas system. Nature Protocols, 9: 2395-2410.
Turkec, A., Lucas, S.J., Karacanli, B., Baykut, A., Yuksel, H., 2016. Assessment of a direct hybridization microarray strategy for comprehensive monitoring of genetically modified organisms (GMOs). Food Chemistry, 194: 399-409.

Van den Elzen, P.J.M., Townsend, J., Lee, K.Y., Redbrook, J.R., 1985. A chimaeric hygromycin resistance gene as a selectable marker in plant cells. Plant Molecular Biology, 5(5): 299-302.

Vijayakumar, K.R., Martin, A., Gowda, L.R., Prakash, V., 2009. Detection of genetically modified soya and maize: Impact of heat processing. Food Chemistry, 117(3): 514-521.

Weeks, D.P., Spalding, M.H., Yang, B., 2016. Use of designer nucleases for targeted gene and genome editing in plants. Plant Biotechnology Journal, 14(2): 483-495.

Xu, R.F., Li, H., Qin, R.Y., Li, J., Qiu, C.H., Yang, Y.C., Ma, H., Li, L., Wei, P.C., Yang, J.B., 2015. Generation of inheritable and "transgene clean" targeted genome-modified rice in later generations using the CRISPR/Cas9 system. Scientific Reports, 5: 1-10.

Yuzbasioglu, G., Yilmaz, S., Marakli, S., Gozukirmizi, N., 2016. Analysis of Hopi/Osr27 and Houba/Tos 5/Osr13 retrotransposons in rice. Biotechnology \& Biotechnological Equipment, 30(2): 213-218

Zhang, H., Zhang, J., Wei, P., Zhang, B., Gou, F., Feng, Z., Mo, Y., Yang, L., Zhang, H., Xu, N., 2014. The CRISPR/Cas9 system produces specific and homozygous targeted gene editing in rice in one generation. Plant Biotechnology Journal, 12(6): 797-807.

Zhang, M., Yu, Y., Gao, X., Zhang, K., Luan, F., Zhu, Y., Qu, B., 2015. Event-specific quantitative detection of genetically modified wheat B72-8-11 based on the 3' flanking sequence. European Food Research and Technology, 240(4): 775-782.

Zuraida, A.R., Rahiniza, K., Zulkifli, A.S., Alizah, Z., Zamri, Z., Aziz, A., 2013. Hygromycin as selective marker in Agrobacterium-mediated genetic transformation of indica rice $\mathrm{MR}_{219}$. Journal of Tropical Agriculture and Food Science, 41(1): 7179. 